\title{
Structural basis of glycan specificity in human rotaviruses
}

\author{
Liya Hu${ }^{1}$, Wilhelm Salmen², Banumathi Sankaran ${ }^{3}$, Sasirekha Ramani², Mary K. Estes², \\ B. V. Venakataram Prasad ${ }^{1,2}$
}

${ }^{1}$ Verna and Marrs McLean Department of Biochemistry and Molecular Biology, ${ }^{2}$ Department of Molecular Virology and Microbiology, Baylor College of Medicine, Houston, TX, ${ }^{3}$ Berkeley Center for Structural Biology, Lawrence Berkeley National Laboratory, Berkeley, CA.

Initial attachment to host cells is a critical event in the life cycle of a virus. This involves recognition by the viruses of specific receptors on the cell surface, including glycans. Viruses typically exhibit strain-dependent variations in recognizing specific glycan receptors, a feature that contributes significantly to cell tropism, host specificity, host adaptation and interspecies transmission. Rotaviruses (RVs) are the leading cause life-threatening gastroenteritis in infants and young children worldwide. Glycan recognition by RVs for cell attachment is mediated by the distal VP8* domain of the spike protein VP4. Although structurally well conserved with a galectin-like fold, sequence-wise, VP8* is the least conserved among RV structural proteins giving to rise to a phylogeny consisting of $\sim 50 \mathrm{P}$ genotypes which can be broadly grouped in 5 clades. While some animal RVs bind to sialo-glycans, most human RVs recognize histo-blood group antigens (HBGAs), which are the determinants of blood types. To elucidate the molecular mechanisms of how human RVs including sporadic, globally-dominant, and neonate-specific strains recognize specific cellular glycans, we have carried out glycan array, crystallography, and cellular biology studies. Glycan array analysis supported by infectivity assays show that human RVs bind to specific HBGAs, such as A-type, H-type and precursor HBGAs in a genotype-dependent manner. Our crystal structures of VP8* of human RVs in complex with HBGAs reveal remarkable variations in the glycan binding sites providing a possible structural basis for the global dominance, neonate-specificity, and interspecies transmission exhibited by human RVs. This work is supported by grants from NIH and Robert Welch Foundation. 